\title{
An Experimental Study on the Simple Active Noise Control Windows
}

\author{
Wongeun $\mathrm{Oh}^{1}$
}

\begin{abstract}
The noise reduction ability of simple duct-type active window systems that use the ANC(active noise control) technology is demonstrated with scale model room experiments. The advantages of the duct-type active windows compared to traditional multi-channel systems are less computational complexity and no interference between channels. In this paper, we examine the noise reduction performance of the simple active window system using a scale-model. The results show that the system can reduce effectively the power of transmitted noise through the window.
\end{abstract}

Keywords - Active Noise Control, Active Windows, Active Window Systems

\section{INTRODUCTION}

SINCE the urban populations have regularly increased, a S large percent of the population are being suffered from the traffic noise. Such noises can be effectively reduced by closing windows that have good sound insulation quality. However, this solution only works when the windows are closed and is less effective in summer time or the tropical countries.

This problem might be solved with active noise control(ANC) technology that is recognized as an effective technique for reducing low frequency noise [1], [2]. Many active window systems have been studied to reduce the noise transmission through an open window [3]-[8]. Huang et al. investigated the active window system that was constructed by staggering the opening sashes of a spaced double glazing window to allow ventilation and natural light. An analytical model based on the modal expansion method was developed to calculate the low frequency sound field inside the window and the room and to be used in the active noise control simulations [3]. Hu et al. used a transparent thin film speaker in a home window that could provide both an invisible audio playback and an active noise cancellation device. The developed system was evaluated in a scaled cabin, and the results showed that the developed system could preserve the audio sound while canceling external noise [4]. Kwon \& Park proposed an active window system to reduce the exterior noise entering a room through an open window. The performance of the system was verified in a scale-model experiment for directional noise sources, and the results showed that the overall average sound pressure level inside the scale model was reduced by as much as $10 \mathrm{~dB}$ in the frequency band of 400-1000 Hz [5]. Pàmies et al.

Wongeun $\mathrm{Oh}^{1}$ is with Sunchon National University, Suncheon-si, Jeonnam, Korea. presented an experimental work on active control of sound transmission through a restricted opening bottom hinged window and its application to aircraft fly-over incident noise. The result showed that an increase of almost $3 \mathrm{~dB}$ of transmission loss in the low frequency range which is equivalent to a reduction of $50 \%$ in the loss of insulation caused by opening the window [6]. However, in spite of the promising results, many practical issues arise when the active windows have applied to real applications, i.e. the computational complexity and the performance degradation by the interference between loudspeakers. These problems are mainly caused by the use of the multi-channel algorithm.

In this paper, a single channel duct-type active window system [7], [8] that reduces the noise transmitted to the room is examined by scale-model experiments. The system is a short length duct that configured to cancel the noise from the outside to the room using a single input single output feedforward algorithm. The control complexity of the active window system is reduced to the simple active control problem in a duct that is relatively well established and the interference problem does not arise because of the single control loudspeaker. The feasibility of the proposed system was examined in scale-model experiments.

\section{PROPOSED ACTIVE WINDOW SYSTEMS}

Several issues should be considered to implement the ANC technology to the open windows, i.e. the performance of the noise reduction, the complexity of the controller, the interference between loudspeakers, and the practical design architecture.

The single channel duct type active window systems might alleviate such practical problems [7]. The systems use single-input single-output structure and the single channel algorithm. The advantages of the systems are that they have no interference between channels, relatively simple structure, and less computational complexity than the previously developed active window systems.

A cross section sketch of the simple active window system installed in a room is shown in Fig. 1. 


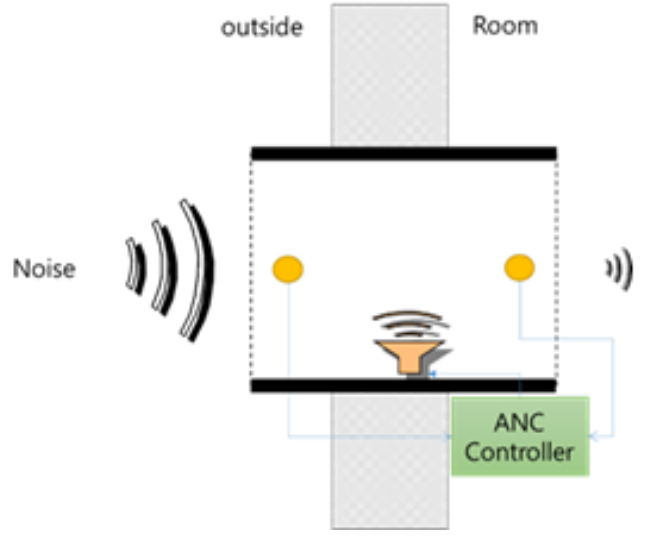

Fig. 1 Sketch of the proposed ANC window

The system consists of a short length duct, a reference microphone, an error microphone, and a control loudspeaker. A reference microphone measures the exterior noise and sends it to the controller. Then the controller calculates 180 degrees out of phase signal, and the loudspeaker plays it. This makes the destructive interference between the incident noise and the control sound. Therefore, the sound pressure levels in the room caused by the exterior noise passing through the window can be decreased. The error microphone picks up the residual noise and provides feedback to the controller so as to optimize the performance of the ANC system.

Since the proposed system has a duct shape and single channel structure, it gives several advantages than the other active windows systems.

1) The controller might be free of the causality problem because the noise travels relatively long path from outside to the room.

2) Instead of the multichannel approach, the single input single output algorithm can be used for reducing the room noise caused from the exterior sources.

3) It provides direct view and natural ventilation (the dwellers can feel the natural wind).

\section{EXPERIMENTAL SETUP}

The scale-model experiments are conducted to examine the performance of the simple active windows. The experimental setup is shown in Fig. 2. The dimension of the model room is 0.9 $\mathrm{m} \times 1.2 \mathrm{~m} \times 0.9 \mathrm{~m}$. The walls are made of $3 \mathrm{~cm}$ thick PVC panels and covered with $2.5 \mathrm{~cm}$ thick sound absorbing materials. The dimension of the window is $0.12 \mathrm{~m} \times 0.19 \mathrm{~m}$, and the duct type active window (length $0.52 \mathrm{~m}$ ) is installed on it.

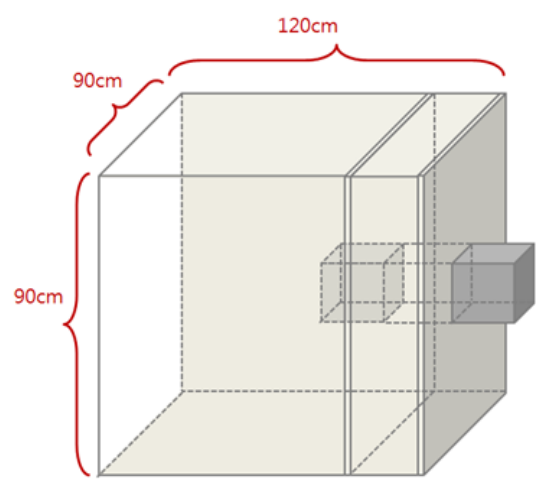

Fig. 2 Experimental setup of the duct-type ANC window

The ANC controller used to the experiment is a 'Demo S-Fan 90' ANC system by Silentium [9]. It acts as a duct-type active window that is suitable for testing the feasibility of the proposed system. The S-Fan system consists of two microphones(one is a virtual microphone), two control loudspeakers, and an adaptive controller embedded with a single channel feedforward ANC algorithm. Fig. 3 shows a photograph of the Demo S-Fan.

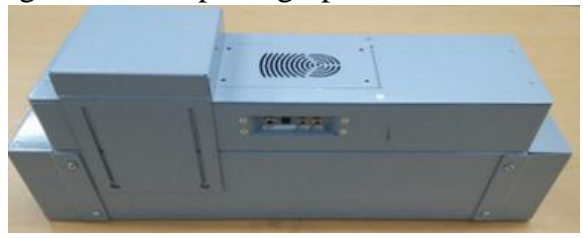

Fig. 3 Photograph of the Demo S-Fan

For monitoring and analyzing purposes, Silentium's monitor program and analyzing program were used. The Monitor program is an interface between the laptop and the controller, and the S-ANC Tool is for analyzing the data acquired during the calibration process.

Fig. 4 shows the photograph of the overall experimental system. The primary noise was generated by Yamaha MSP5 monitoring loudspeaker located in front of the window, and two measurement microphones (Earthworks M30S and Audix TR40A) were used for recording the sound levels and the spectrum changes of the noise in the room.

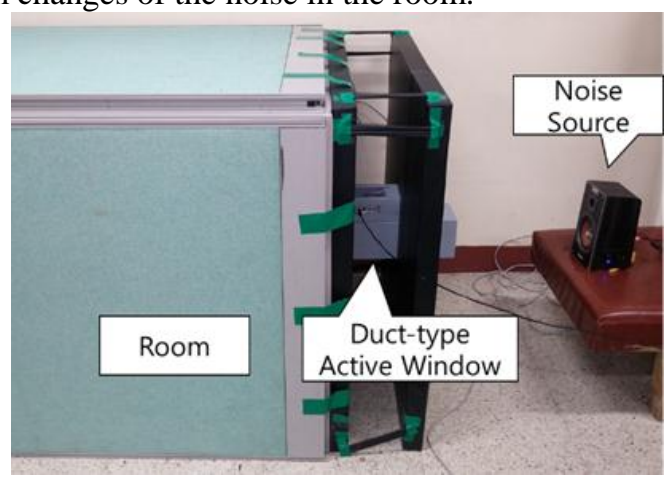

Fig. 4 Photograph of the experimental setup

\section{RESULTS}

Two types of experiments were conducted to verify that the proposed active window system can reduce the noise 
transmission through the open window; (1) playing pink noise and music while the back panel was closed for simulating small-size rooms; (2) repeat but the back panel was opened to examine large-size rooms.

Table 1 lists the averaged sound pressure level (SPL) at the observation point in the room when back panel was closed. When the ANC was On, the averaged reduced sound pressure level was $6.4 \mathrm{dBA}$ for pink noise and $9.4 \mathrm{dBA}$ for music. Fig. 5 shows the spectrum of the noise measured in the room with and without ANC.

TABLE I

THE AVERAGE SPL IN THE ROOM WHEN THE BACK PANEL WAS CLOSED.

\begin{tabular}{llll}
\hline & ANC Off & ANC On & Difference \\
\hline Pink noise & $68.2 \mathrm{dBA}$ & $61.8 \mathrm{dBA}$ & $6.4 \mathrm{dBA}$ \\
Music & $69.9 \mathrm{dBA}$ & $60.5 \mathrm{dBA}$ & $9.4 \mathrm{dBA}$ \\
\hline
\end{tabular}

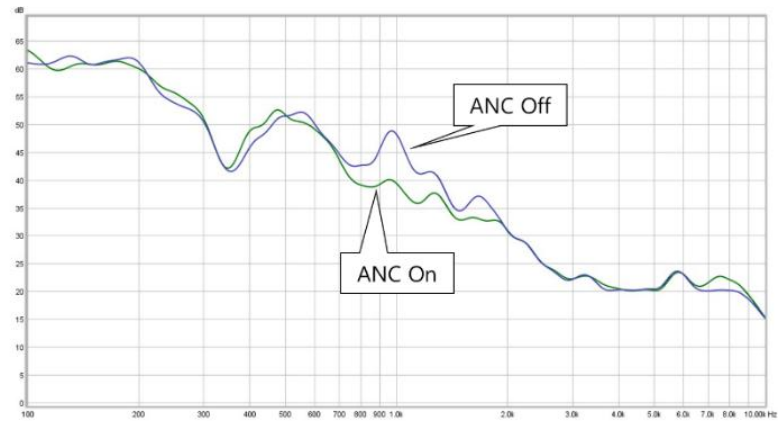

Fig. 5 Averaged spectrum at the error microphone with and without ANC.

The experiment showed that the proposed active window can reduce the transmitted noise when the window was open. However, there are several factors that affect the performance of the active windows. For example, the size of the room, the room geometry, and the window location can reduce or increase the noise level at the particular position in the room. However, it is difficult to analyze the relationship between those factors and the ANC performance, since the sound field in the room is quite complex and highly nonlinear.

To investigate the effect of the room size on the ANC performance, another experiment was conducted while the back panel was opened (See Fig. 6). The results of the averaged sound pressure level at the observation point in the room are presented in Table 2. The averaged SPL difference between ANC Off and ANC On is $5.1 \mathrm{dBA}$ for pink noise and $3.8 \mathrm{dBA}$ for music. The performance of the ANC window was slightly degraded when the back panel was open. It means that we need more control power to reduce the noise when the room is large comparing to the window.

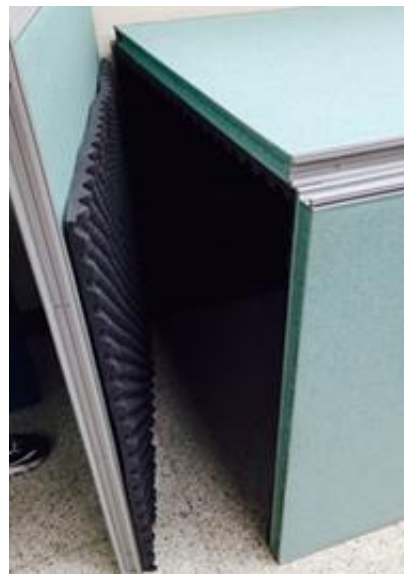

Fig. 6 The back panel of the scale-model room

TABLE II

THE AVERAGE SPL IN THE ROOM WHEN THE BACK PANEL OPEN.

\begin{tabular}{llll}
\hline \hline & ANC Off & ANC On & Difference \\
\hline Pink noise & $67.1 \mathrm{dBA}$ & $62.0 \mathrm{dBA}$ & $5.1 \mathrm{dBA}$ \\
Music & $66.2 \mathrm{dBA}$ & $62.4 \mathrm{dBA}$ & $3.8 \mathrm{dBA}$ \\
\hline
\end{tabular}

\section{CONCLUSION}

In this paper, the feasibility of the simple duct-type ANC window has been demonstrated experimentally by a scaled model. Two experiments were conducted; (1) playing pink noise and music while the back panel was close in order to simulate small-size rooms; (2) repeat but the back panel was open to examine large-size rooms. The result shows that the simple active window system can reduce the room noise level caused by the exterior sources. However, for practical applications, additional studies should be conducted, i.e. the optimal window size for the room volume and the effect of room parameters on the ANC performance.

\section{ACKNOWLEDGMENT}

This research was supported by Basic Science Research Program through the National Research Foundation of Korea(NRF) funded by the Ministry of Education (NRF-2014R1A1A2057077).

\section{REFERENCES}

[1] S. J. Elliott and P. a. Nelson, "Active noise control," IEEE Signal Process. Mag., vol. 10, no. 4, 1993. http://dx.doi.org/10.1109/79.248551

[2] S. M. Kuo and D. R. Morgan, Active Noise Control Systems. John Wiley \& Sons, Inc., 1996.

[3] H. Huang, X. Qiu, and J. Kang, "Active noise attenuation in ventilation windows.,” J. Acoust. Soc. Am., vol. 130, no. 1, pp. 176-188, Jul. 2011. http://dx.doi.org/10.1121/1.3596457

[4] S. Hu, R. Rajamani, and X. Yu, "Invisible speakers in home windows for simultaneous auxiliary audio playback and active noise cancellation," Mechatronics, vol. 22, no. 8, pp. 1031-1042, Dec. 2012. http://dx.doi.org/10.1016/j.mechatronics.2012.07.003

[5] B. Kwon and Y. Park, "Interior noise control with an active window system,” Appl. Acoust., vol. 74, no. 5, pp. 647-652, May 2013. http://dx.doi.org/10.1016/j.apacoust.2012.11.005 
[6] T. Pàmies, J. Romeu, M. Genescà, and R. Arcos, "Active control of aircraft fly-over sound transmission through an open window," Appl. Acoust., vol. 84, pp. 116-121, 2014.

http://dx.doi.org/10.1016/j.apacoust.2014.02.018

[7] W. Oh, "Noise reduction in rooms using a single channel active noise controller," in Engineering \& Technology, Computer, Basic \& Applied Sciences, Bangkok, 2016.

[8] W. Oh, "Performance Evaluation of Active Windows Using Speech Intelligibility," in Int. Conf. on Engineering Technologies and Big Data Analytics, Bangkok, 2016

[9] Silentium LTD., "Silentium's Demo S-Fan 90." [Online]. Available: http://www.silentium.com/products/demo-s-fan-90/. 